frequency of sleep disturbance at baseline, among participants with or at risk for radiographic knee $\mathrm{OA}(\mathrm{ROA})$, and to estimate the association between the presence of inflammation on knee MRI and pain that disturbs sleep among knees that developed ROA.

Methods: Knees from the Osteoarthritis Initiative (OAI) with or at risk for ROA were included in this longitudinal analysis. Self-reported frequency of restless sleep in the past week was assessed using a CES-D question. Knee symptoms were assessed annually using the WOMAC. Knees that developed incident ROA (i.e., $\geq 2$ Kellgren-Lawrence grade, KLG) through four years of follow-up were assessed for effusion-synovitis on non-contrast-enhanced 3T MRI using the MRI Osteoarthritis Knee Score (MOAKS) from annual clinic visits between four years prior to incident ROA and up to one year after ROA detection. Effusionsynovitis represents a combination of joint effusion and synovial thickening on fluid-sensitive sequences and scored as 0 (normal), 1 (mild), 2 (moderate), or 3 (severe). Annual mean WOMAC total score was estimated using a mixed model with participant and knee treated as random effects, in 5,028 knees at risk for ROA at baseline, and in 3,893 knees with ROA at baseline. Log-binomial regression with generalized estimating equations was used to estimate the association between effusion-synovitis and knee pain in bed that disturbs sleep, adjusted for age, sex, and BMI in a sample of 355 knees with an average of $3.8 \mathrm{MRI}$ assessments.

Results: There was dose-dependent effect, with participants reporting restless sleep 1-2 days, 3-4 days, and 5-7 days in the past week having higher mean WOMAC total scores compared to those who reported $<1$ day of restless sleep (i.e., difference in means: 2.5 [95\% Cl: 1.3 to 3.8 ], 5.1 [95\% Cl: 3.2 to 7.1 ], and 10.1 [95\% Cl: 7.6 to 12.6 ], respectively) among knees with ROA ( $\mathrm{KLG} \geq 2$ ) at baseline. Differences in average WOMAC total score between groups were relatively persistent over nine years (Figure 1). A similar dose-dependent effect of restless sleep was observed among knees at risk of ROA (i.e., KLG 0 or 1). Among knees that developed incident ROA, those with mild effusion-synovitis had a $52 \%$ higher risk of knee pain in bed that disturbs sleep at the same visit $(R R=1.52$; $95 \% \mathrm{Cl}: 1.13$ to 2.04 ), while knees that had moderate/severe effusion-synovitis had more than double the risk of knee pain that disturbs sleep ( $R R=2.55 ; 95 \%$ $\mathrm{Cl}: 1.87$ to 3.47 ), compared to knees with no MRI-detected effusion-synovitis.

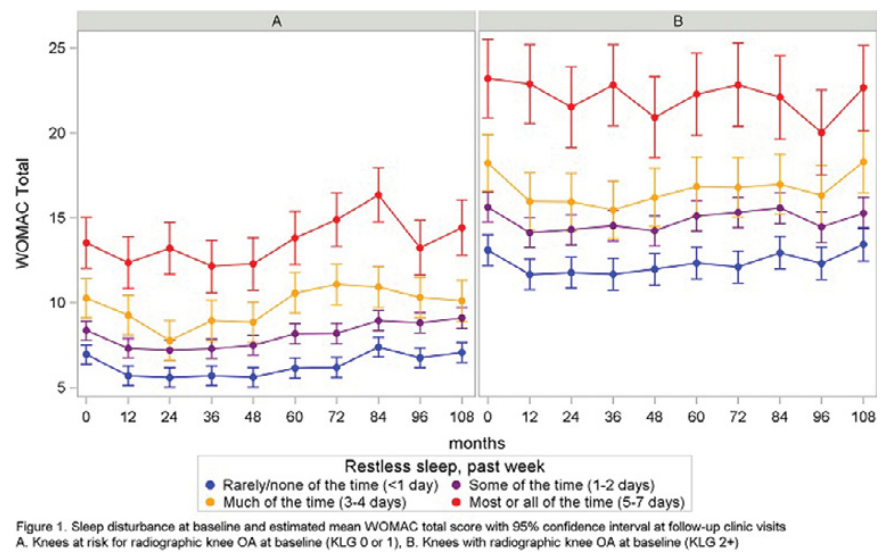

Conclusions: Restless sleep was associated with knee symptoms and disability in a dose-dependent manner, with average levels persistent over nine years of follow-up among knees with and at risk of ROA. Effusion-synovitis was associated with pain that disturbs sleep among knees that developed incident ROA. Sleep disturbance and knee inflammation may be important targets for interventions in knee osteoarthritis.

Disclosure of Interest: C. K. Kwoh Grant/research support from: Abbie, EMD Serono, E. Ashbeck: None declared, A. Guermazi Grant/research support from: Boston Imaging Core Laboratory, Consultant for: MerckSerono, TissueGene, Ortho Trophix, AstraZeneca, Genzyme, F. Roemer Grant/research support from: Boston Imaging Core Laboratory, S. Parthasarathy: None declared DOI: 10.1136/annrheumdis-2017-eular.6273

\section{SAT0501 PREVALENCE OF HAND OSTEOARTHRITIS IN A MODEL OF AUTO-IMMUNE DISEASE, SJÖGREN SYNDROME: RESULTS OF A PROSPECTIVE STUDY}

C. Glanowski ${ }^{1}$, J. Sellam ${ }^{1}$, E. Bergé ${ }^{2}$, R. Seror ${ }^{2}$, A.-L. Tomi $^{3}$, X. Chevalier ${ }^{3}$, F. Berenbaum ${ }^{1}, X$. Mariette ${ }^{2}$, R. Belkhir ${ }^{2} .{ }^{1}$ Rheumatology, Hôpital Saint Antoine, Paris; ${ }^{2}$ Rheumatology, Hôpital de Bicêtre, le Kremlin-Bicêtre; ${ }^{3}$ Rheumatology, Hôpital Henri Mondor, Créteil, France

Background: Despite the role of chronic low-grade inflammation in osteoarthritis $(\mathrm{OA})$, prevalence of $\mathrm{OA}$ during inflammatory or auto-immune diseases remains poorly assessed. A single study without a valid control group [1] and daily practice suggest an increased frequency of hand OA (HOA) in primary Sjögren Syndrome (pSS).

Objectives: We aimed investigate the prevalence of radiographic, symptomatic or erosive HOA in pSS and to compare these results with age and sex-matched control population suffering from dry eyes and mouth without auto-immune manifestation (sicca patients).

Methods: We included women with pSS fulfilling 2002 AECG European-American criteria and control women suffering from sicca symptoms without autoimunity who underwent a multidisciplinary day hospital. Standardized radiographs of hands were performed and were all read by an experimented reader. Radiographic HOA was defined as Kellgren-Lawrence score $\geq 2$ on at least one joint. Erosive HOA was defined using the Verbruggen scoring system (phase E, subchondral erosion or R, remodelling of subchondral plate). Symptomatic HOA was defined as radiographic $\mathrm{HOA}$ associated with spontaneous hand joint pain and/or with a FIHOA $\geq 5$ at the time of radiographs. We compared pSS versus sicca patients, pSS with radiographic $\mathrm{HOA}$ (pSS+, $\mathrm{HOA}+$ ) versus $\mathrm{pSS}$ without radiographic $\mathrm{HOA}$ (pSS+, HOA-), and finally pSS with radiographic $\mathrm{HOA}(\mathrm{pSS}+, \mathrm{HOA}+$ ) versus sicca patients with radiographic $\mathrm{HOA}$ (pSS-, $\mathrm{HOA}+$ ).

Results: We included 34 patients with pSS (median age [range] 54.5 [28-84] years) and 54 patients with only sicca symptoms (57 [24-85] years). Among pSS patients, $41 \%$ had radiographic HOA, $12 \%$ had symptomatic $\mathrm{HOA}$ and $9 \%$ had erosive HOA. These prevalences did not differ from sicca patients $(52 \%$ radiographic HOA $p=0.45,28 \%$ symptomatic $\mathrm{HOA} p=0.11,9 \%$ erosive $\mathrm{HOA}$ $p=1.0$ ). The trend for a higher prevalence of symptomatic HOA in sicca patients compared to pSS is expected since these patients frequently suffer from dryness associated with fibromyalgia. In pSS patients, radiographic HOA was associated with a higher average age ( $p S S+\mathrm{HOA}_{+}: 63,5$ versus $\mathrm{pSS}+\mathrm{HOA}-: 48,5$ years old, $p<0,01$ ), with menopause (pSS+HOA+: $100 \%$ versus $p S S+H O A-: 45 \%, p<0,01$ ) and hypothyroidism (pSS+HOA+: $64 \%$ versus $p S S+H O A-: 25 \%, p=0.04$ ). There was no pSS feature such as autoantibodies profile or pSS disease activity, associated with radiographic or symptomatic HOA. Finally, HOA symptoms did not differ between patients with pSS and sicca symptoms. The prevalence of radiographic and erosive HOA in pSS and non-autoimmune sicca group seems in the same range as that observed in women from Framingham's cohort $(44 \%$ and $10 \%$ respectively). And prevalence of symptomatic HOA seems similar in the pSS (12\%) and in women from Framingham's cohort (16\%).

Conclusions: The prevalence of $\mathrm{HOA}$ is not increased in pSS, a model of auto-immune diseases. Patients with pSS have the same HOA risk factors than in general population (age and menopause) and also hypothyroidism that may be involved in OA pathophysiology.

\section{References:}

[1] Aksoy and al, J Rheumatol 2016.

[2] Mariette and al, Rheumatology (Oxford) 2003.

[3] Haugen and al, Ann Rheum Dis 2011.

Acknowledgements: Osteoarthritis group of French Society of Rheumatology.

Disclosure of Interest: None declared

DOI: 10.1136/annrheumdis-2017-eular.2476

\section{SAT0502 A RANDOMIZED, PLACEBO-CONTROLLED, PROOF-OF-CONCEPT EFFICACY STUDY OF A MICROSOMAL PROSTAGLANDIN E SYNTHASE-1 (MPGES1) INHIBITOR AND A PROSTAGLANDIN E RECEPTOR (EP4) ANTAGONIST IN THE TREATMENT OF CANINE OSTEOARTHRITIS PAIN}

C. Robertson-Plouch ${ }^{1}$, J.R. Stille ${ }^{2}$, P. Liu ${ }^{1}$, S. Malcolm², D. Brown ${ }^{3}$, M. Warner ${ }^{1}$, M.J. Fisher ${ }^{1} .{ }^{1}$ Lilly Research Laboratories; ${ }^{2}$ The Chorus Group, Ell Lilly and Company, Indianapolis; ${ }^{3}$ School of Veterinary Medicine, University of Pennsylvania, Philadelphia, United States

Background: Inflammation is a known contributor to osteoarthritis (OA) pain in animals and humans. While translation of human treatments to companion dogs is common, translation from companion dogs to humans is less frequent. We present results of a clinical study, in client-owned canines with moderate OA pain, which evaluated the efficacy of 2 molecules targeting the actions of prostaglandin E, either by modulating its production (mPGES1 inhibitor; LYA), or subsequent pharmacology through 1 of its 4 receptors (EP4 antagonist, LYB).

Objectives: To provide translational and comparative data to inform the potential utility of each of these mechanisms for treatment of chronic OA pain in humans and dogs.

Methods: A multicenter, randomized, double-blind, placebo (PBO)- and activecontrolled trial in client-owned canine patients with moderate $\mathrm{OA}$ pain in $\geq 1$ hindlimb/forelimb joint. Dogs $\geq 2$ years of age with Liverpool Osteoarthritis in Dogs (LOAD) Mobility total score $\geq 13$ to $<46$ were randomized (1:1:1:1) to 2 weeks of LYA (1.5 mg/kg/day), LYB $(25 \mathrm{mg} / \mathrm{kg} /$ day), carprofen $(4.4 \mathrm{mg} / \mathrm{kg} /$ day), or PBO. Efficacy versus PBO was assessed by mean change from baseline (CFB) to Week 2 in the Canine Brief Pain Inventory (CBPI) Pain Interference (PI) Score (primary endpoint), and for secondary endpoints: CBPI Pain Severity (PS) and Overall Impression (OI) subscores, and LOAD Mobility score. Data were analyzed by mixed-effect model for repeated measures with treatment, time, and interaction of treatment and time as fixed effects, and with baseline score, site, and weight as covariates. Posterior probability of treatments being superior to PBO was calculated with Bayesian methods.

Results: Of 163 dogs randomized, 158 (96.9\%) completed the study. Treatment arms were well-balanced for baseline characteristics (mean [standard deviation] age: 9.3 [3.0] years, weight: $54.6 \% 15-32 \mathrm{~kg}, 45.4 \%>32-50 \mathrm{~kg}$, CBPI PI: 5.1 [2.1]; CBPI PS: 4.2 [1.9]; LOAD Mobility: 24.1 [5.6]). Improvements (CFB) in CBPI PI were observed in all treatment groups after 2 weeks (Table). For LYA, 